\title{
Oblique Drilling by Ti:sapphire fs Laser in Silicon
}

\author{
Anett GÁRDIÁN ${ }^{* 1}$, Judit BUDAI ${ }^{* 1}$, Miklós FÜLE ${ }^{* 2}$ and Zsolt TÓTH ${ }^{* 1,3}$ \\ ${ }^{* 1}$ Department of Optics and Quantum Electronics, University of Szeged, H-6720 Szeged, Dóm tér 9., \\ Hungary \\ ${ }^{* 2}$ High Intensity Laser Laboratory, Department of Experimental Physics, University of Szeged, $H$ - \\ 6720 Szeged, Dóm tér 9., Hungary
${ }^{* 3}$ Department of Oral Biology and Experimental Dental Research, University of Szeged, H-6720 \\ Szeged, Tisza Lajos krt. 64., Hungary \\ E-mail: miklos.fule@gmail.com
}

Femtosecond laser ablation results in sharp edges and high aspect ratio holes in case of most solid materials. In this work it is shown that oblique drilling is possible by oblique illumination even in high thermal conductivity materials. Experiments were performed on silicon by varying the number and the angle of the incident laser pulses of a Ti:sapphire laser. Three phases of the formation of the oblique holes were distinguished and described: 1. A cone-like hole is formed in the direction of laser irradiation. 2. The bottom of the hole is widened. 3. The shape of the ablated hole becomes tube-like. It is shown, that reflections from the inner walls have an influence on the shape of the holes. Two models were set up to calculate energy distribution in conical and tube-like holes to describe the hole formation.

DOI: $10.2961 / \mathrm{jlmn} .2015 .01 .0015$

Keywords: Femtosecond laser ablation, oblique drilling, hole formation, pulse propagation, reflection

\section{Introduction}

Femtosecond ablation became widely used both for basic research and for industrial applications. Comparative studies [1-3] have demonstrated that fs laser ablation has advantages over nanosecond ablation. These are: higher precision, reduced heat affected zone, burr-free edges and smaller amount of debris around the ablated spot. This allows one to produce high aspect ratio holes with very high precision and reproducibility using compact laser systems [4-10]. Only a few studies took tilted samples or oblique illumination [11-13] into account.

In this paper, we provide a detailed analysis of oblique drilling produced by fs laser of silicon in air atmosphere. Silicon target was selected due to its importance in many industrial applications. We particularly investigated the influence of tilt angle and number of pulses on the depths of the holes induced by fs laser pulses.

\section{Experimental}

In our experiments a Ti:sapphire laser was applied as the source of the laser pulses. The central wavelength was $800 \mathrm{~nm}$ and the pulse length was $30 \mathrm{fs}$ at $144 \mathrm{~Hz}$ repetition rate. The energy at the sample surface was kept in the experiments at $477 \mu \mathrm{J}$. The beam of the Ti:sapphire laser was directed by a mirror onto the sample and it was focused by a $85 \mathrm{~mm}$ focal length lens. The focal point was positioned on the surface of the tilted sample. The number and the angle of the incident laser pulses were varied. A schematic draw of the experimental setup can be seen in Fig. 1.

For test material silicon was chosen, because it has high thermal conductivity: $149 \mathrm{~W} \cdot \mathrm{m}^{-1} \cdot \mathrm{K}^{-1}$ at room temperature. Before irradiation the (100) silicon wafer pieces were cleaned in ultrasonic bath under acetone and ethanol separately. Then the Si pieces were mounted on a 4-axis universal stage, which allowed the tilt of the sample.

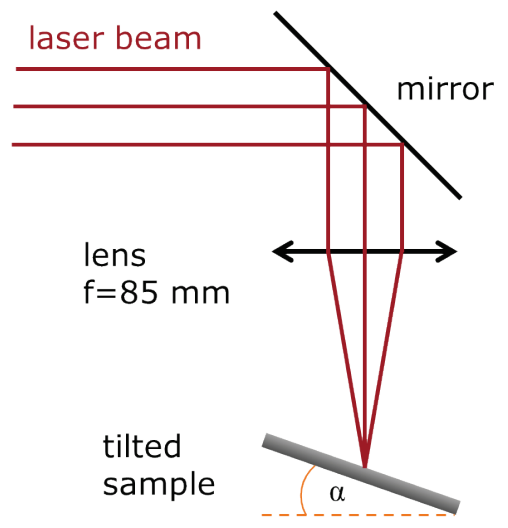

Fig. 1 The applied experimental setup for oblique illumination of the samples.

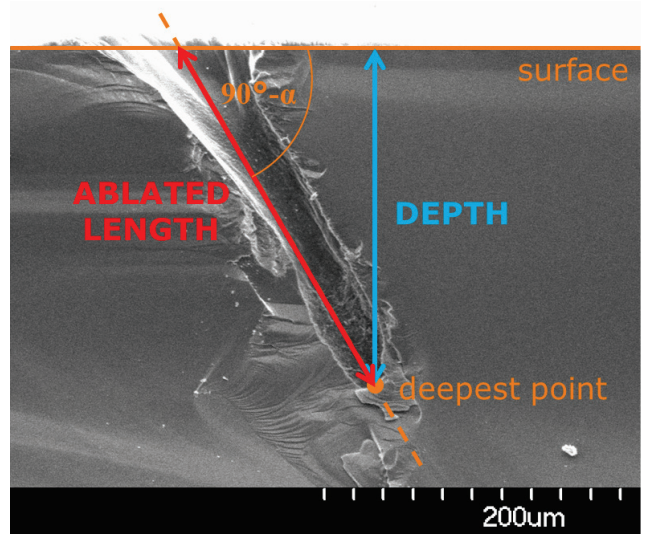

Fig. 2 The measurement of depth and ablated length. 

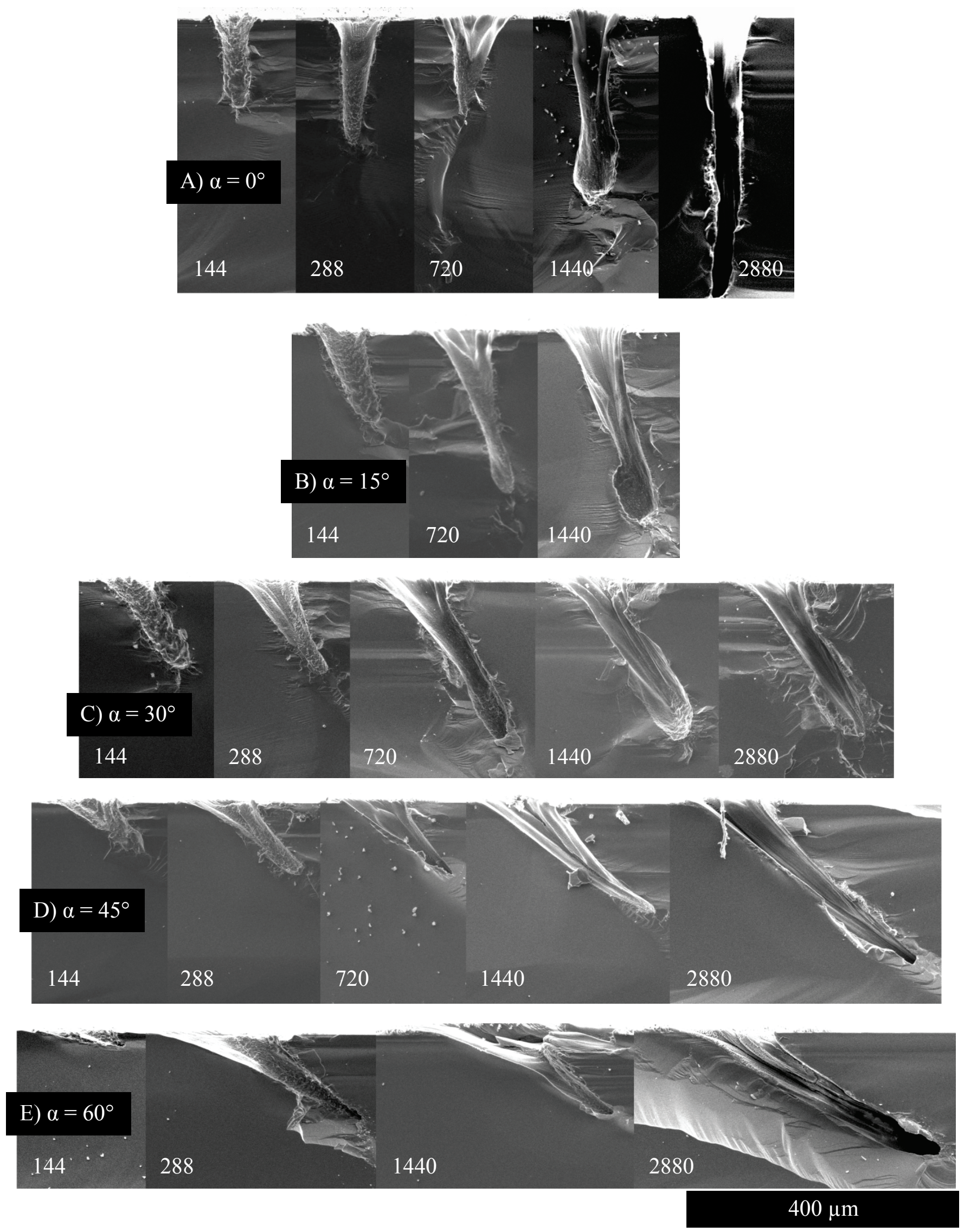

Fig. 3 The drilled holes at different angles of incidence (A-E) by different number of laser pulses (indicated at the lower left corner of the images). 
The top view of the ablated holes was investigated by Nikon Optiphot 100S optical microscope. For cross-section investigations the samples were sectioned and scanning electron microscope (SEM, Hitachi S-4700) images were recorded. Measurements were performed on the images by ImageJ [14]: In the followings depth is defined as the distance between the sample surface and the deepest point of the drilled hole. The angle of incidence $(\alpha)$ fairly well determines the angle of the holes relative to the surface: $90^{\circ}-\alpha$. Setting a line at this angle, that is going through the deepest point of the hole, the distance between this deepest point and the intersection of the line with the surface plane gives the ablated length (Fig. 2). As the sectioning of the samples was not perfect, the cross-sectional plane does not coincide exactly with the fracture plane. Therefore the measurement results of ablation depths and lengths have uncertainty that cannot be estimated accurately.

\section{Results and discussion}

In the SEM images (Fig. 3) it can be seen that the shape of the holes drilled by low and high pulse numbers differs from each other at each angle of incidence. The shapes, corresponding to the same pulse numbers, are similar even at different angles of incidence. Initially, as a result of ablation by a few hundreds of shots, cone-like hole formation can be observed. After 700-1000 shots widening appears at the end of the hole. Drilling by some thousands of laser pulses results in tube-like holes. At deeper regions narrowing of the holes occurs.

In Fig. 4 the length of the drilled holes is plotted as a function of shot number. The different angles of incidence are marked with symbols. Although the uncertainty is large, the length of the holes increases in the same way, monotonously with increasing pulse number. The ablation length seems to be independent of the angle of incidence.

In Fig. 5 the depth of the end of the holes is plotted as a function of shot number. The measured depth decreases with the increase of the angle of incidence. It can be clearly seen from the figure, that depths, corresponding to higher angles of incidence, have lower depth values. According to the system geometry and as a consequence of the definitions the depth equals the ablation length multiplied by $\cos \alpha$.

A logarithmic type curve can be fitted on the measured points of ablation length. As there is a (large) error in the measured results the fitted curve is only informational. The derivative of the fitted curve gives the ablation rate which is plotted as a continuous curve in Fig 6. In Fig. 6 the ratio of the ablation length and the corresponding pulse number is also plotted as a function of the number of laser pulses. Comparing this to the ablation rate curve there is a similarity between their tendencies. At low pulse numbers $(<500)$ the values of the ablation rate curve and the measurements results both are determined by the fluence. At large pulse numbers the average ablation length/pulse includes the larger ablation lengths of the previous laser pulses, too, so it has higher value than the ablation rate.

The conditions of oblique hole drilling are determined by the size of the laser spot, the optically excited region and the thermally excited region. Usually, when cw lasers are applied or high thermal diffusivity samples are processed by ns pulse length lasers, then the size of the thermally affected zone largely exceeds the optical penetration depth. In this way the application of oblique illumination does not result in holes, that exactly follow the direction of the focused beam. Thermal diffusion becomes negligible, when using fs lasers or materials with low thermal diffusivity. As a consequence, the direction of material removal is determined by the spatial intensity distribution, i.e. it follows the direction of the laser pulses.

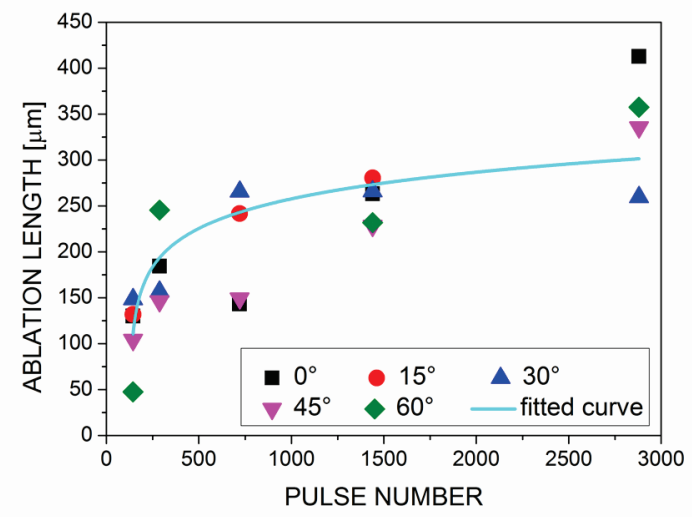

Fig. 4 The ablation length as a function of pulse number for different angles of incidence. The fitted curve indicates a logarithmic tendency in the ablation rate.

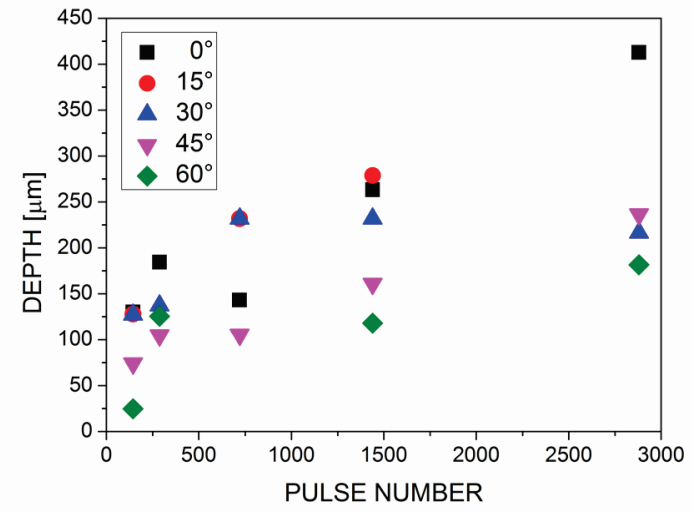

Fig. 5 The pulse number dependence of depth at different angles of incidence.

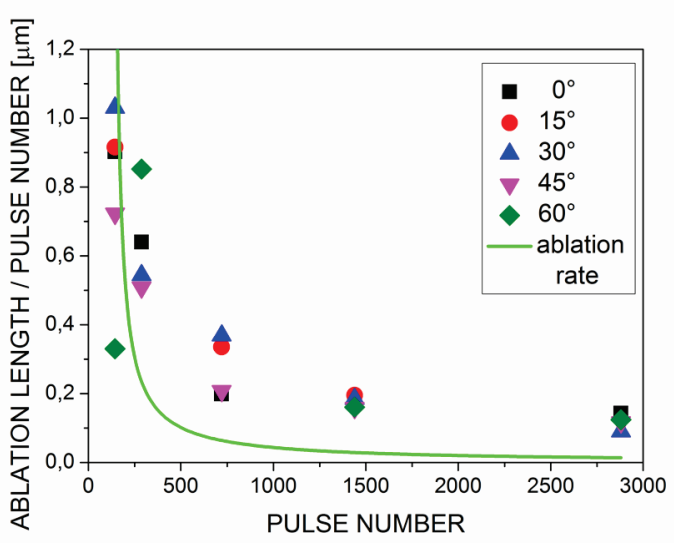

Fig. 6 Comparison of the ablation length/pulse values to the ablation rate as a function of pulse number. 

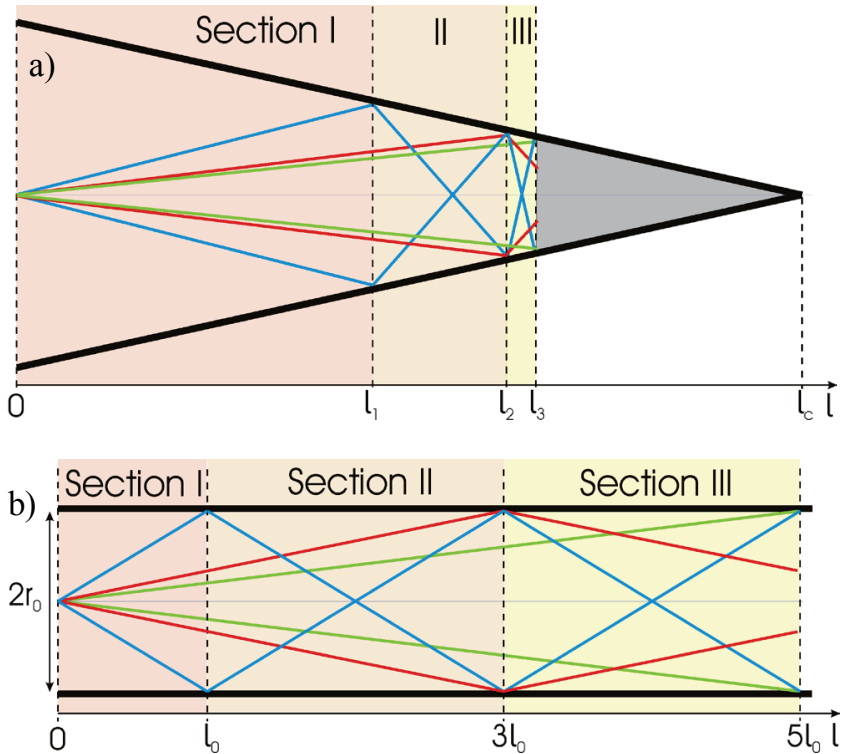

Fig. 7 Schematic draws of the cone-like (a) and the tubelike (b) hole models. The outer rays diverging from the focal point is marked by blue, the inner ones by red and green. For better visualization the diameter-length ratio is larger, than in real case. The borders of the sections (at $\mathrm{l}_{0}$, $\left.l_{1}, l_{2}, l_{3}\right)$ are also indicated by dashed lines. See text for detailed description.

More precisely, the excitation lengths have to be distinguished for low and high intensities, which result in different ablation speeds [15]. In the case of mild ablation thermal effects are negligible at low intensities; the ablation is governed by the optical penetration into the material. In the case of strong ablation, even in the case of fs lasers, above certain threshold intensity, thermal evaporation becomes effective, since high temperature molten surfaces evaporate until the laser excited region cools down. The amount of the removed material can be further increased by the explosive removal of the overheated liquid in the form of mixture of gas and nanoparticles. The appearance of ionic, atomic and nanoparticle parts follow each other, as indicated by pump-probe measurements [16].

There are three phases of the hole formation: 1 . In the first phase a cone-like hole is formed due to the laser irradiation. The direction of the cone is determined by the angle of incidence. 2. For further laser pulses the bottom of the hole gets wider. 3. At high number of laser pulses the shape of the hole is more similar to tube than cone.

The first pulses ablate the smallest surface; therefore in this case the intensity is the highest, which leads to the appearance of strong ablation. Both optical excitation and thermal effects contribute to material removal. The ablation rate of these pulses is the highest as the focal point is placed on the surface. As the hole forms, due to the ablation, the illuminated surface increases in the hole. This leads to the decrease in intensity, especially at the curved parts, and at the walls of the holes. Therefore ablation becomes mild at the walls of the holes, and there the ablation speed decreases quickly. As a result, those surface elements are ablated with the highest speed, which are perpendicular to the direction of the laser beam, namely the bottom of the hole. Therefore a cone-like hole forms in the direction of the laser beam.
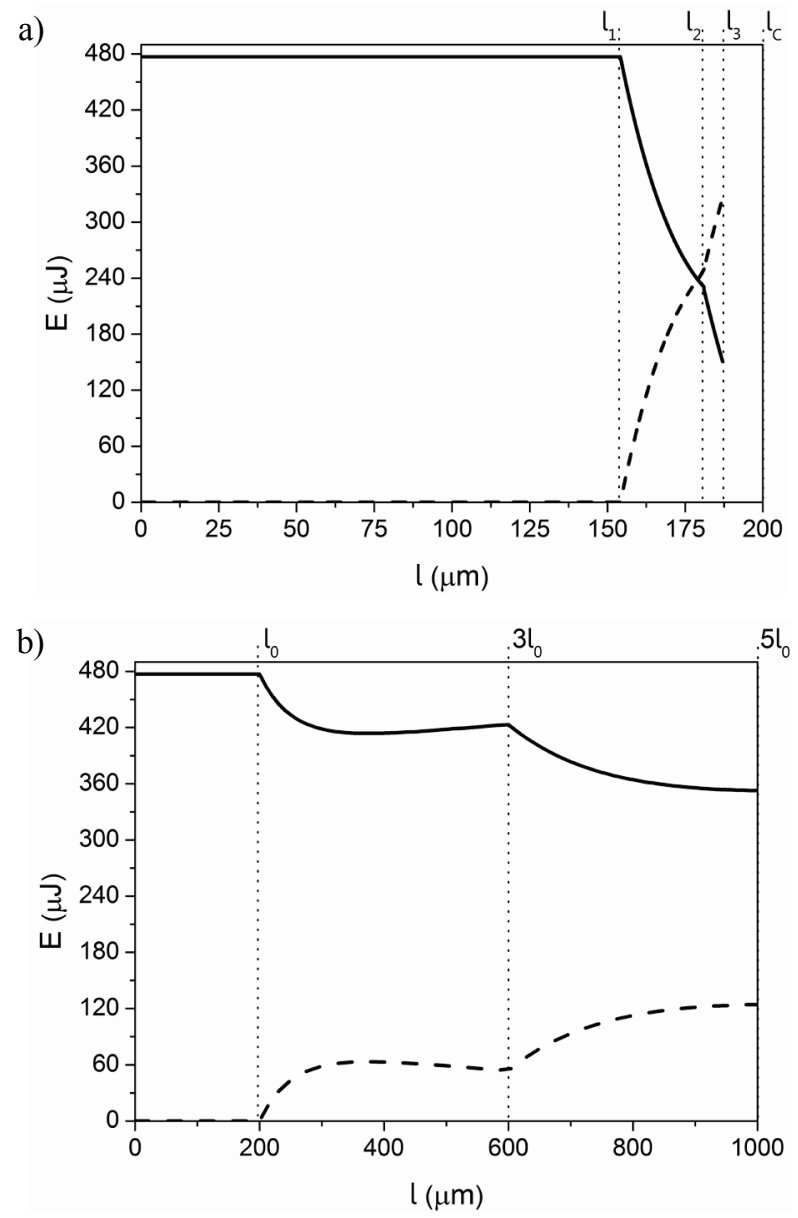

Fig. 8 The result of the models: the cone-like (a) and the tube-like (b) hole. In both cases the solid line refers to the energy value in the hole, while the dotted line represents the total energy coupled into the wall as a function of the length of the hole. The borders of the sections (at $l_{0}, l_{1}, l_{2}$, $l_{3}$ ) are also indicated by dashed lines. See text for detailed description.

Calculations on the hole shape were performed by Kuan-Chung Yao and Jehnming Lin [12] for the irradiation of graphite using 1 pulse of Nd:YAG laser (1064 nm, pulse duration $10 \mathrm{~ms}$ ).

The calculation of fluence at the walls of the ablated holes can be applied in our case in the same way. Note, that in [12] the pulse duration is much longer, but thermal conductivity of graphite is much lower, in elevated temperatures it becomes negligible.

The fluence at the bottom of the holes depends on their length, the divergence of the focused laser beam and the reflectance on the sidewalls of the drilled hole. During the propagation of the diverging laser beam in the hole multiple reflections occur at the walls. This can cause secondary ablation, if the local fluence exceeds the threshold fluence value.

Two simple models were set up to describe energy distribution along the holes, which affects the shape during hole formation for the 2. and 3. phases. The first one refers to the cone-like holes (Fig. 7 a), while the second one is for describing the tube-like period (Fig. 7 b). In both cases the rays diverge in a cone from the focal point which is placed on the axe of symmetry. All parameters, the divergence of 
the laser pulses, the position of the focal point, the apex angle of the cone and the radius of the tube were set to the experimental details. The models for normal incidence and for oblique drilling are the same as the holes are formed in the direction of laser irradiation. It is assumed that the intensity distribution is homogeneous. The absorption of the plasma was not taken into consideration. The cone and the tube were divided into three parts in length relative to the positions where the outer rays reach the wall of the tube directly and after reflections. In section I there are no reflections, the rays are diverging, in section II the outer rays reflected 1 time; in section III the second reflection of the outer rays occurs. In Figs. 7 a) and b) and in Figs. 8 a) and $b)$ the $l$ axis is the distance from the entrance of the hole. The positions of the section borders are marked with dashed lines on this axis. These sections correspond to image formation of caleidoscopes. In caleidoscopes the multiple images are due to multiple reflections in the inner sides of tubes or prisms. The calculations, based on the geometrical parameters, were performed in the three sections separately. The angular dependence of the reflections was taken into account. For other focusing conditions than ours the change in spot size and beam divergence can be taken into account by setting the appropriate model parameters as cone length, apex angle and tube diameter.

The results of the conical-shaped hole model can be seen in Fig. 8 a). $\sim 70 \%$ of the pulse energy is coupled into the walls before the end of the third section $\left(\mathrm{l}_{3}\right)$. This means that in sections II and III widening appears. As the hole gets wider, the angle of incidence on that part of wall changes pulse by pulse, it increases. The reflectivity increases owing to the higher angles of incidence, which result in further decrease in absorbed energy. As a consequence, more energy reaches the deeper parts of the hole, it gets deeper and its shape is no more cone-like, instead it is like a tube.

In the case of tube-like hole the energy coupling on the sidewalls is much lower than in the other model because of the higher angle of incidence. The fluence, or the effective pulse energy inside the hole is increased by the reflected rays. More energy reaches the bottom of the hole so deepening of the hole is more characteristic than widening. However, widening also appears where the diverging and once reflected rays all reach the walls. The calculations also show slight increase in fluence in this region, especially the end of section II (Fig. 8 b)). During the ablation of the bottom of the hole material is removed. The ablation plume is deposited on the sidewalls and on the sample surface, too, which was detected by optical microscopic observations. As a consequence, both widening or narrowing of the sidewalls can happen during a dynamic process, depending on whether ablation or deposition is dominant. According to the experimental results narrowing is more typical at the deeper parts of the hole. The main reason of this is the decrease in the fluence as the hole gets longer. The final diameter value is established when material removal from the walls and deposition equilibrates. As a final result of these processes high aspect ratio, deep, inclined holes are formed, even in high thermal conductivity materials.
In Fig. 9 there is the summary of the three steps of laser drilling. The initial laser pulses cause strong ablation (red curves). Firstly a shallow hole is formed, which modifies the fluence distribution. This leads to the formation of a tilted conical hole. At some hundred laser pulses mild ablation occurs (orange curves) as the fluence decreases on the increased steepness walls. At the bottom of the hole the angle of incidence is low; and the ablation gets stronger by the effect of reflected rays, so there the hole is widened. At the widened surfaces the angle of incidence increases and more light is directed to the bottom. As the hole is deepened the ablated material is deposited on the walls. For some thousand pulses the shape of the hole becomes tubelike (yellow curve).

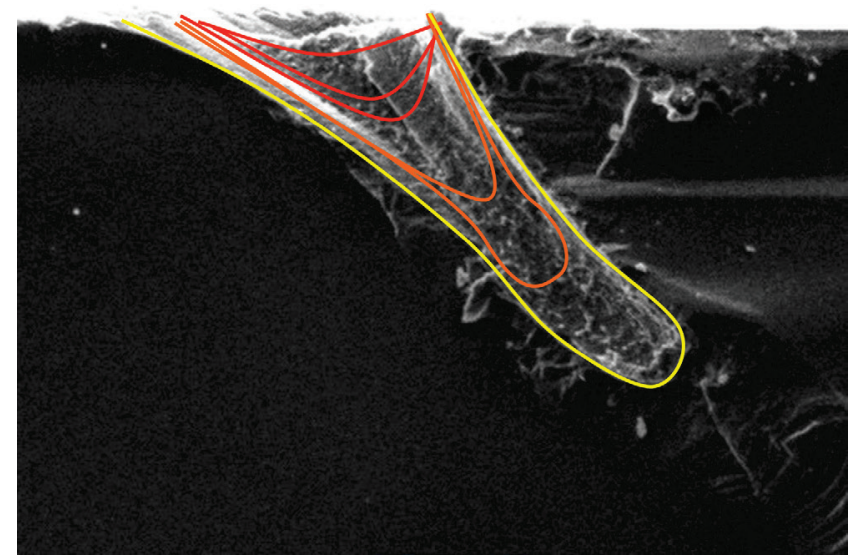

Fig. 9 Schematic draw of the steps of drilling.

\section{Conclusions}

Multiple fs laser pulses allow drilling high aspect ratio holes even in high thermal conductivity materials. In this study we have shown that the direction of the holes follows the beam path which makes possible to drill tilted or oblique holes. The process starts with a high fluence strong ablation. As the hole forms intensity is decreased in a higher amount close to the borders than in the center. This nonuniform fluence distribution leads to a hole formation with a conical form. The next step is the widening of the bottom of the hole because of multiple reflections in the cone and the resulting increase in the angle of incidence at the walls which is a result of this geometry. The final shape of the hole becomes tube-like when the inner reflections lead the reflected light to the bottom of the ablated hole. Narrowing of the hole occurs at the bottom due to the decrease in fluence and deposition on the sidewalls.

Deep hole drilling in metals or semiconductors in directions determined by a laser beam may have an importance in forming via holes between microelectronic structures or in fabricating micro channels for microfluidic applications.

\section{Acknowledgment}

The project was partially funded by TÁMOP-4.2.2.A11/1/KONV-2012-0047- "New functional materials and their biological and environmental answers" and is supported by the European Union and co-financed by the European Social Fund. 


\section{References}

[1] B.N. Chichkov, C. Momma, S. Nolte, F. von Alvensleben and A. Tünnermann: Appl. Phys. A 63, (1996) 109.

[2] A. Semerok, C. Chaleard, V. Detalle, J.-L. Lacour, P. Mauchien, P. Meynadier, C. Nouvellon, B. Salle, P. Palianov, M. Perdrix and G. Petite: Appl. Surf. Sci., 138-139, (1999) 311.

[3] R. Le Harzic, N. Huot, E. Audouard, C. Jonin, P. Laporte, S. Valette, A. Fraczkiewicz and R. Fortunier: Appl. Phys. Lett., 80, (2002) 3886.

[4] S. Karimelahi, L. Abolghasemi, P.R. Herman: Appl. Phys. A 114, (2014) 91.

[5] S. Döring, S. Richter, H. Heisler, T. Ullsperger, A. Tünnermann and S. Nolte: Appl. Phys. A, 112, (2013) 623.

[6] C.H. Wang, L.T. Zhang, Y.S Liu, G.H. Cheng, Q. Zhang and K. Hua: Appl. Phys. A, 111, (2013) 1213.

[7] S. Döring, J. Szilagyi, S. Richter, F. Zimmermann, M. Richardson, A. Tünnermann and S. Nolte: Optics Express, 20, (2012) 27147.
[8] P.J. Ding, Q.C. Liu, X. Lu, X.L. Liu, S.H. Sun, Z.Y. Liu, B.T. Hu and Y.H. Li: Nucl. Inst. Methods in Phys. Res. B, 286, (2012) 40.

[9] S. Ahn, D.J. Hwang, H.K. Park and C.P. Grigoropoulos: Appl. Phys. A, 108, (2012) 113.

[10] S. Döring, S. Richter, A. Tünnermann and S. Nolte: Apl. Phys. A, 105, (2011) 69.

[11] C.Y. Wu, C.W. Shu and Z.C. Yeh: Opt. Las. Eng., 44, (2006) 842.

[12] K.C. Yao and J. Lin: Opt. Las. Tech., 48, (2013) 110.

[13] J. Kamalu, P. Byrd and A. Pitman: J. Mat. Proc. Tech., 122, (2002) 355

[14] W.S. Rasband: ImageJ, U.S. National Institutes of Health, Bethesda, Maryland, USA, http://imagej.nih.gov/ij/, 1997-2014.

[15] S. Nolte, C. Momma, H. Jacobs, A. Tünnermann, B.N Chichkov, B. Wellegehausen and H. Welling: J. Opt. Soc. Am. B, 14, (1997) 2716.

[16] N. Jegenyés, J. Etchepare, B. Reynier, D. Scuderi, A. Dos-Santos and Z. Tóth: Appl. Phys. A, 91, (2008) 385.

(Received: July 14, 2014, Accepted: January 11, 2015) 